\title{
An outbreak of Coxsackievirus A6- associated hand, foot, and mouth disease in a kindergarten in Beijing in 2015
}


Lijuan Chen ${ }^{2}$, Quanyi Wang ${ }^{2^{*}}$ and Yan He

\begin{abstract}
Background: Coxsackievirus A6 (CVA6) is one of the major agents to cause hand, foot and mouth disease (HFMD) outbreaks globally. The objective of this study is to investigate the epidemiologic and clinical manifestations of CVA6 outbreak, and thus guide the diagnosis and treatment of the disease, as well as disease prevention.

Methods: An HFMD outbreak in a kindergarten was reported to Shijingshan District Center for Disease Control and Prevention (SCDC) on November 2, 2015 in Beijing, China. Epidemiological investigation was conducted. We performed a nine-week follow-up study to collect and analyze the clinical manifestations of HFMD cases.

Results: The outbreak yield 56 (15.7\%) clinical diagnosed HFMD cases out of 357 registered children in the kindergarten with the mean age of 3.5 years old. This outbreak lasted for three days and ceased after initiating infectious disease controlling procedures, including periodical suspension of the kindergarten activities, environmental disinfection, and family health education. Fifty-one cases were followed for nine weeks. The positive rate of clinical manifestations of rash, fever, desquamation, pigmentation and onychomadesis were $100.0 \%, 84.3 \%, 68.6 \%, 17.6 \%$ and $43.1 \%$, respectively. Children developed desquamation within the first 4 weeks after disease onset and developed onychomadesis between the 3th and 8th week after disease onset. Children with desquamation had 9.3 (95\%Cl: 1.836-47.437) times higher odds of developing onychomadesis compared to those without this manifestation. Ten out of 14 collected samples were CVA6 positive, and five positive samples shared a high degree of similarity in the VP1 nucleotide and amino acid sequences (99.9-100.0\% and 100\%).

Conclusion: This HFMD outbreak was caused by CVA6, featured with delayed symptoms. Emerging CVA6associated HFMD and its delayed symptoms should be paid more attention to reduce outbreaks and provide more information to doctors and parents.
\end{abstract}

Keywords: Hand foot and mouth disease, Disease outbreak, Coxsackievirus A6, Onychomadesis

\section{Background}

Hand, foot and mouth disease (HFMD) is an infectious disease characterized by clinical symptoms of vesicular rashes and stomatitis of the oral mucosa affecting patients' hands, feet and occasionally the buttocks. The most

\footnotetext{
* Correspondence: bjcdcxm@126.com; heyanlb2014@163.com

${ }^{\dagger}$ Jie Li and Rong Zhu contributed equally to this work.

${ }^{2}$ Institute for Infectious Disease and Endemic Disease Control, Beijing Center for Disease Prevention and Control, No.16, Hepingli Middle Street, Beijing 100013, People's Republic of China

'Department of Epidemiology and Biostatistics, School of Public Health, Capital Medical University, No. 10 Xitoutiao, You'anmen Wai, Fengtai District, Beijing 100069, People's Republic of China
}

susceptible patient population of HFMD is children less than 5 years old. HFMD is caused by viruses belonging to the species Enterovirus A or B, genus Enterovirus, family Picornaviridae, among which, human enterovirus 71 (EV-A71) and Coxsackievirus A16 (CVA16) were the most prevalent pathogens in previous publications [1-3]. Only EV-A71 is almost exclusively associated with severe disease.

From 2012 onward, Coxsackievirus A6 (CVA6) has been reported as an emerging pathogen of the HFMD epidemic globally. In 2013, the etiological surveillance system in Beijing and other cities in China identified a

(c) The Author(s). 2018 Open Access This article is distributed under the terms of the Creative Commons Attribution 4.0 International License (http://creativecommons.org/licenses/by/4.0/), which permits unrestricted use, distribution, and reproduction in any medium, provided you give appropriate credit to the original author(s) and the source, provide a link to the Creative Commons license, and indicate if changes were made. The Creative Commons Public Domain Dedication waiver (http://creativecommons.org/publicdomain/zero/1.0/) applies to the data made available in this article, unless otherwise stated. 
sudden rise of CVA6 positive rate, which turned out to be one of the predominant pathogens of HFMD [4-7]. Since then, CVA6 has spread across mainland China and drawn serious public health attention.

In 2015, there were in total six HFMD outbreaks occurred in the kindergartens in Beijing. The number of cases ranged from 11 to 14 among the five of them, and remaining one had 56 cases. This outbreak occurred in a kindergarten in Shijingshan district in Beijing. There were 357 children and 51 school staffs in this kindergarten. The children were divided and assigned to 11 classes (average class size was 32 ). Daily extra-curricular activities were held, in which children from different classes attended lessons together. We performed a further investigation on this large-scale HFMD outbreak in this kindergarten and found that it was caused by CVA6 infection.

In this study, we aimed to collect and summarize epidemiological and clinical evidence of CVA6 infected HFMD, and thus to support and guide the diagnosis, treatment, prevention and control of the disease.

\section{Methods}

\section{Case definition and outbreak definition}

A clinical case of HFMD was defined as presenting with oral ulcers and maculopapular or vesicular rash distributed over the hands, feet and buttocks, with or without fever. Cases were classified as severe if they presented any cardiopulmonary complications (pulmonary edema, pulmonary hemorrhage, or cardiorespiratory failure) or neurological complications (aseptic meningitis, encephalitis, encephalomyelitis, acute flaccid paralysis, or autonomic nervous system dysregulation) [8].

According to the "Protocol of control practice for the clustered cases and outbreaks of hand foot and mouth disease" issued by the National Health and Family Planning Commission of China in 2012 [9], an HFMD outbreak was defined as $\geq 10$ cases of HFMD occurring in a kindergarten or school within a week. Once an outbreak is reported from a kindergarten or a school, the health practitioners from local CDC will have a risk assessment on the situation, upon which a school suspension decision will be made. In order to confirm the disease causing agent of the outbreak, at least five samples are required to be collected and analyzed.

\section{Sample and data collection}

Once the district CDC got a report of HFMD outbreak, the epidemiological investigation was conducted immediately. Demographic information (ID, birth date, gender), clinical symptoms (fever, rash, sore throat, desquamation, onychomadesis), and epidemiological data (onset date of HFMD, the date of visiting a hospital, onset date of delayed symptoms, clinical diagnostic result and laboratory test result) and throat swab samples were collected by CDC staffs on the same day of the field investigation with prior written informed consent. The teacher in charge of each classroom was responsible for reporting any new case to the doctors of community healthcare centers who would pay the sick children a home visit, collected information with prior written informed consent, and reported to the local CDC afterwards. Parents or guardians of the sick children were followed up twice by telephone to collect information about the delayed symptoms, such as onychomadesis, desquamation and chromatosis, at 4th and 9th weeks after the onset of HFMD.

\section{Sample processing mode, nucleotide extraction and virus identification}

The specimens were vortexed for $1 \mathrm{~min}$ and centrifuged at $4000 \times \mathrm{g}$ for $20 \mathrm{~min}$ before nucleotide extraction. Total nucleotide extraction was performed with MagNA Pure LC Total Nucleic Acid Isolation Kit-Large Volume (ROCHE, Co, USA) by a Roche MagNA Pure LC $2.0 \mathrm{nu}$ cleic extraction system (ROCHE, Co, USA) according to the manufacturer's instructions. EV, EV-A71 and CVA16 were identified using real-time RT-PCR Kit (DAAN Gene, Guangzhou, China) [10]. Samples of non-EV-A71 and non-CVA16 were further identified by detecting the VP1 region according to previously described method [11]. Complete nucleotide sequences of VP1 genes were amplified using previously described specific primers [12]. Polymerase Chain Reaction (PCR) products of complete VP1 genes were purified and sequenced using ABI PRISM310 Genetic Analyzer.

\section{Phylogenetic analysis of CVA6}

To further study the homology of sequences from viruses in this outbreak, sequence analysis of VP1 was carried out using Molecular Evolutionary Genetics Analysis Version 6.0 (MEGA6). The phylogenetic tree was constructed by Maximum likelihood method with bootstrapping of 1000 replicates. A total of 5 CVA6 isolates representing this outbreak were aligned and compared with the corresponding region of CVA6 isolates from HFMD casess through 2009 to 2015 in Beijing.

\section{Statistical analysis}

Statistical analysis was conducted using SPSS17.0 software (IBM SPSS Inc., Chicago, IL, USA). Continuous variables in asymmetric distribution were described using the median with 25th and 75th percentiles. Frequencies and proportions were used for categorical variables. Comparisons of proportions were performed using the Chi-square test. A two-tailed $p$ value of less than 0.05 (2-sided significance testing) was considered to be statistically significant. 


\section{Ethics statement}

This study was in compliance with the Helsinki Declaration and was approved by the Human Research Ethics Committee of Beijing CDC. The approval number of the local ethics committee was 201,809 . Sample collection in this study was agreed by the patient's guardian with prior written informed consent.

\section{Results}

In 2015, a total of six outbreaks of HFMD occurred in Beijing kindergartens (Table 1). Four out of six outbreaks were caused by CVA6. The remaining two outbreaks were caused by EV71 and CVA16. Among the six outbreaks, the largest one was caused by CVA6, in which 56 cases were identified.

\section{Epidemiological investigation}

This outbreak occurred in a kindergarten in Shijingshan district and was reported on November 2, 2015. In response, Shijingshan district CDC (SCDC) initiated an epidemiological investigation immediately.

From October 26th to November 5th, a total of 56 clinically diagnosed cases from 10 classes were reported. The attack rate was $15.7 \%$ (56/357). From October 27th to 30th, 16 children from different classes were reported with fever or rash on body. During the following weekend (October 31 and November 1), this outbreak attained the peak and a total of 22 cases were obtained. From November 2nd to 5th, another 17 cases were reported (Fig. 1).

There were 357 children and a health-care doctor in this kindergarten. Every morning, school doctor did regular body checks for each child to see if they present any fever, skin rashes or herpangina. Once a symptom is found, the parents or guardians would be notified to take their children for further medical examination. During the noon break, the same regular body checks would be performed again. The first case in this outbreak appeared on October 26, 2015 a 5-year-old girl who had low-grade fever with no rash on her hands or feet. She felt ill in the afternoon and was taken home by her mother and rested at home for following days. On October 29, her mother found generalized vesicular rashes distributed over her hands and feet and took her to a hospital, where she was diagnosed with HFMD. Her parents did not recall or report exposure to any HFMD cases before diagnosis.

\section{Outbreak control measures}

Infection-control measures, including case isolation, contact medical observation, environmental disinfection, suspension of the kindergarten, and health education, were immediately implemented. All the children diagnosed with HFMD were isolated at home for at least 10 days until recovered. Kids could return to kindergarten only if the community health care center proved that they have completely recovered. Classrooms, indoor playground, bathrooms, kitchens and recreation facilities were cleaned and disinfected thoroughly. Based on the fact that children from 10 out of the 11 classes were infected (according to the clinical diagnosis) and HFMD was prone to spread rapidly, the kindergarten was advised to take a periodical suspension for 10 days. The health practitioners from local CDC and health care centers did health education for children and their parents, as well as the kindergarten staffs.

\section{Epidemiological characteristics, clinical manifestations and laboratory test result}

During this outbreak, to obtain more detailed information about the prognosis and the delayed symptoms such as desquamation and onychomadesis, we contacted all the HFMD patients in this the outbreak. Five parents of the patients refused, thus, 51(91.1\%) volunteered to enroll in this study and were successfully followed up at 4th and 9th week after illness onset. Among the 51 patients, $26(51.0 \%)$ were girls, and the median age was 44 months (IQR 41.0-51.0). Six out of 51 cases (11.8\%) had a history of HFMD disease.

All the cases sought for medical treatment and were clinically diagnosed as mild HFMD in seven hospitals and fully recovered without any complications. Epidemiology and clinical presentations of HFMD cases in this study were shown in Table 2 . The most common clinical manifestation was skin rash $(51 / 51,100.0 \%)$, followed by fever $(43 / 51,84.3 \%)$. A total of 35 children $(68.6 \%, 35 / 51)$

Table 1 Epidemiology characteristics of six HFMD outbreaks in Beijing in 2015

\begin{tabular}{lllllllll}
\hline ID & District & Classification & Number of Cases & Attack rate (\%) & ${ }^{a}$ Age (year) & Male/Female & Date & Agent \\
\hline 1 & Fangshan & Kindergarten & 14 & 17.7 & $3.0(2.0,3.0)$ & $2.5: 1$ & Apr & CVA16 \\
2 & Mentougou & Kindergarten & 16 & 6.5 & $4.0(4.0,5.0)$ & $1.3: 1$ & Jun & CVA6 \\
3 & Fangshan & Kindergarten & 22 & 12.0 & $3.0(3.0,3.0)$ & $1.8: 1$ & Jul & EV-A71 \\
4 & Fengtai & Kindergarten & 11 & 2.1 & $5.0(5.0,5.0)$ & $0.8: 1$ & Jul & CVA6 \\
5 & Chaoyang & Kindergarten & 14 & 4.0 & $4.0(4.0,4.3)$ & $0.8: 1$ & Sep & CVA6 \\
6 & Shijingshan & Kindergarten & 56 & 15.7 & $3.4(3.7,4.3)$ & $0.9: 1$ & Oct & CVA6 \\
\hline
\end{tabular}

Presented as the median (p25, p75) 


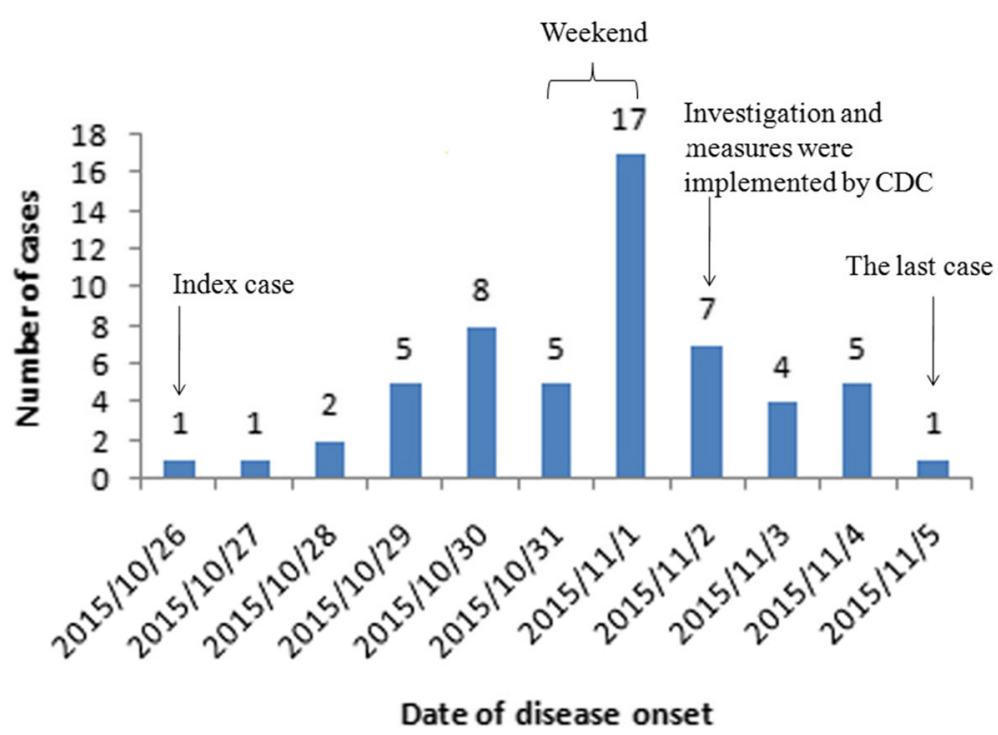

Fig. 1 Number of children with HFMD in the outbreak by date of disease onset $(n=56)$

developed desquamation within the 1st and the 4rd week after disease onset. Of the 51 patients, 22 (43.1\%) experienced onychomadesis between the 3rd and 8th week after disease onset (Additional file 1). The most common location of onychomadesis on the hands and feet were the thumbs $(17 / 51,33.3 \%)$ and halluces $(8 / 51,15.7 \%)$, respectively. In total, 68 finger nails from 22 kids and $17 \mathrm{ft}$ nails from 8 children were found with onychomadesis. Twelve $(54.5 \%, 12 / 22)$ cases showed the symptom of onychomadesis on symmetrical location. Among the 51 patients, two (3.9\%) lost all their fingernails.

Laboratory results were available among 26 cases which were collected by staffs from the health care community center during home visit. The median WBC count, median CRP value, median lymphocyte count and median lymphocyte percentage of cases were $9.2^{*} 10^{9} / \mathrm{L}$ (6.8-10.8), 9.4 mg/L (8.0-13.5), 3.1*10 $/ \mathrm{L}(2.3-4.0)$ and $36.0 \%$ (24.6-52.4), respectively. The WBC counts of $26.9 \%$ (7/26), CRP of $37.5 \%(6 / 16)$ and lymphocyte count of $47.6 \%(10 / 21)$ were above the upper limit of the normal range.

Given that onychomadesis was described as a late complication of HFMD and the role of CVA6 in causing onychomadesis was unclear, we attempted to examine factors associated with the manifestations [13, 14]. As shown in Table 3, age, sex, fever, body temperature, pigmentation and median duration from onset to initial diagnosis were not significantly associated with children's onychomadesis status $(P>0.05)$. Cases with the symptom of desquamation have 9.3 times higher odds (OR $=9.3,95 \% \mathrm{CI}, 1.836-47.437)$ of experiencing onychomadesis comparing with those cases without desquamation.

\section{Pathogen detection and phylogenetic analysis}

During the outbreak, parents of 14 cases (including the parents of the index case) agreed to provide samples for pathogen detection. A total of 14 throat samples from patients were analyzed, and $10(71.4 \%)$ samples (including the sample from the index case) were CVA6 positive. Another three samples, including a swab throat from the health-care teacher and two samples from the tableware and the toilet, were CVA6 negative. Among five out of ten CVA6 positive samples, the VP1 sequences were successfully amplified. The similarity in the nucleotide and amino acid sequences among them was 99.9$100.0 \%$ and $100 \%$, respectively. We analyzed the VP1 of CVA6 viruses from this outbreak (MG488204, MG488205, MG488206, MG488207, and MG488208) and from previous cases during 2009-2015 in Beijing. All the CVA6 strains were classified into five major groups (A-E) (Fig. 2). CVA6 isolates in Beijing from 2009 to 2012 were classified as group D. CVA6 isolates from 2013 to 2015 were classified as group E. The nucleotide identity for CVA6 strains in this study and from 2009 to 2012, 2013-2014 and 2015 in Beijing was $87.7-89.2 \%, 97.8-99.3 \%$ and $94.2-98.3 \%$, respectively. CVA6 strains (including the CVA6 strain from the index case) from the outbreak in this study were all classified as group $\mathrm{E}$.

\section{Discussion}

According to the etiology surveillance system of Beijing, in 2009, 2011, 2012, and 2014, CVA16 was identified as the main pathogen of HFMD, while in 2010, EV-A71 was the major pathogen. However, in 2013 and 2015, CVA6 surpassed CVA16 and EV-A71 to become the 
Table 2 Epidemiology and clinical presentations of HFMD cases in this study

\begin{tabular}{|c|c|}
\hline Characteristics & $\mathrm{N}(\%)$ \\
\hline \multicolumn{2}{|l|}{ Sex } \\
\hline Male & 25(49.0) \\
\hline Female & $26(51.0)$ \\
\hline a Age(months) & $44(41.0-51.0)$ \\
\hline Fever & $43(84.3)$ \\
\hline 'Duration(day) & $1.5(1.0-2.0)$ \\
\hline${ }^{\mathrm{a}}$ The highest temperature $\left({ }^{\circ} \mathrm{C}\right)$ & $38.7(38.4-39.2)$ \\
\hline$\geq 39^{\circ} \mathrm{C}$ & $12(23.5)$ \\
\hline \multicolumn{2}{|l|}{ Rash } \\
\hline Hand & $48(94.1)$ \\
\hline Foot & $37(72.5)$ \\
\hline Oral cavity & $32(62.7)$ \\
\hline Perioral area & 19(37.3) \\
\hline Buttock & $16(31.4)$ \\
\hline Extremity & $6(11.8)$ \\
\hline Trunk & $5(9.8)$ \\
\hline Bullous eruption & 2(3.9) \\
\hline Pigmentation & $9(17.6)$ \\
\hline Desquamation & $35(68.6)$ \\
\hline Onychomadesis & $22(43.1)$ \\
\hline Hands & $21(41.2)$ \\
\hline Thumb & 17(33.3) \\
\hline Forefinger & 10(19.6) \\
\hline Middle finger & $8(15.8)$ \\
\hline Ring fingers & $5(9.8)$ \\
\hline Little finger & $3(5.9)$ \\
\hline Feet & $8(15.7)$ \\
\hline The big toe & $8(15.7)$ \\
\hline The second toe & $1(2.0)$ \\
\hline The third toe & $1(2.0)$ \\
\hline aMedian duration from onset to diagnosis(day) & $1(0.0-3.0)$ \\
\hline \multicolumn{2}{|l|}{ Laboratory test } \\
\hline${ }^{\mathrm{a} W B C}\left(10^{9} / \mathrm{L}\right)$ & $9.2(6.8-10.8)$ \\
\hline${ }^{\mathrm{a}} \mathrm{CRP}(\mathrm{mg} / \mathrm{L})$ & $9.4(8.0-13.5)$ \\
\hline aLymphocyte count $\left(10^{9} / \mathrm{L}\right)$ & $3.1(2.3-4.0)$ \\
\hline a'Lymphocyte percentage (\%) & $36.0(24.6-52.4)$ \\
\hline
\end{tabular}

WBC, white blood cell; CRP, C-reactive protein

a Presented as the median (p25, p75)

predominant pathogen of HFMD [15]. In our study, CVA6 virus was identified as the dominant causative agent, which is consistent with the epidemic background of HFMD in Beijing during 2015.

Considering the fact that the average incubation period of HFMD ranges from 3 to 7 days [16], and it
Table 3 Factors associated with onychomadesis

\begin{tabular}{|c|c|c|c|c|}
\hline & \multicolumn{2}{|c|}{ Onychomadesis } & \multirow[t]{2}{*}{$\mathrm{OR}(95 \% \mathrm{Cl})$} & \multirow[t]{2}{*}{$p^{a}$} \\
\hline & Yes (\%) & No (\%) & & \\
\hline \multicolumn{5}{|l|}{ Age(month) $(n=51)$} \\
\hline $36-47$ & $16(72.7)$ & 15(51.7) & $0.402(0.123-1.318)$ & 0.128 \\
\hline $48-72$ & $6(27.3)$ & 14(48.3) & & \\
\hline \multicolumn{5}{|l|}{$\operatorname{Sex}(n=51)$} \\
\hline Female & $9(40.9)$ & 17(58.6) & $2.046(0.664-6.311)$ & 0.210 \\
\hline Male & 13(59.1) & $12(41.4)$ & & \\
\hline \multicolumn{5}{|c|}{ Median duration from onset to initial diagnosis(days) } \\
\hline$\leq 2$ & $16(72.7)$ & $16(55.2)$ & $0.462(0.140-1.517)$ & 0.199 \\
\hline$>2$ & $6(27.3)$ & 13(44.8) & & \\
\hline \multicolumn{5}{|l|}{$\operatorname{Fever}(n=51)$} \\
\hline No & $5(22.7)$ & $3(10.3)$ & $0.392(0.083-1.860)$ & 0.228 \\
\hline Yes & $17(77.3)$ & 26(89.7) & & \\
\hline \multicolumn{5}{|l|}{ Temperature $(n=43)$} \\
\hline $37.3 \leq T<39^{\circ} \mathrm{C}$ & 13(76.5) & 14(53.8) & $0.359(0.092-1.399)$ & 0.133 \\
\hline $\mathrm{T} \geq 39^{\circ} \mathrm{C}$ & $4(23.5)$ & $12(46.2)$ & & \\
\hline \multicolumn{5}{|c|}{ Desquamation(n = 51) } \\
\hline Yes & 20(90.9) & 15(51.7) & $9.3(1.836-47.437)$ & 0.003 \\
\hline No & $2(9.1)$ & 14(48.3) & & \\
\hline \multicolumn{5}{|c|}{ Pigmentation $(n=51)$} \\
\hline No & $16(72.7)$ & 26(89.7) & $3.250(0.711-14.851)$ & 0.150 \\
\hline Yes & $6(27.3)$ & $3(10.3)$ & & \\
\hline
\end{tabular}

${ }^{a} P<0.05$ from the $x^{2}$ test was considered to be significant

The bold values means significant difference was obtained between

compared groups

might begin to shed enterovirus prior to symptom onset, we speculated that the index case or someone else was the source of infection in this kindergarten [17].

Kindergartens are a highly susceptible location for HFMD outbreaks because large groups of children with close contact could create an excellent incubation site for HFMD. Children are easily infected and more likely easily to spread this disease [18]. In this outbreak, HFMD rapidly spread across 10 of the 11 classes within 11 days. It ceased after the HFMD control measures were implemented by SCDC. We speculated that at least two factors contributed to this rapid transmission. First, the interaction among children from different classes could accelerate the spread of the agent. Second, according to our previous study, some children can transmit the virus prior to symptom onset [17], and infected patients can excrete the virus for a long time. Given the highly infectious characteristics of HFMD, we strongly suggested that cases should be isolated and that thorough environmental disinfection be performed immediately once a case appeared.

As is known, EV-A71 vaccines are available in mainland China, and the protective efficacies are higher than 


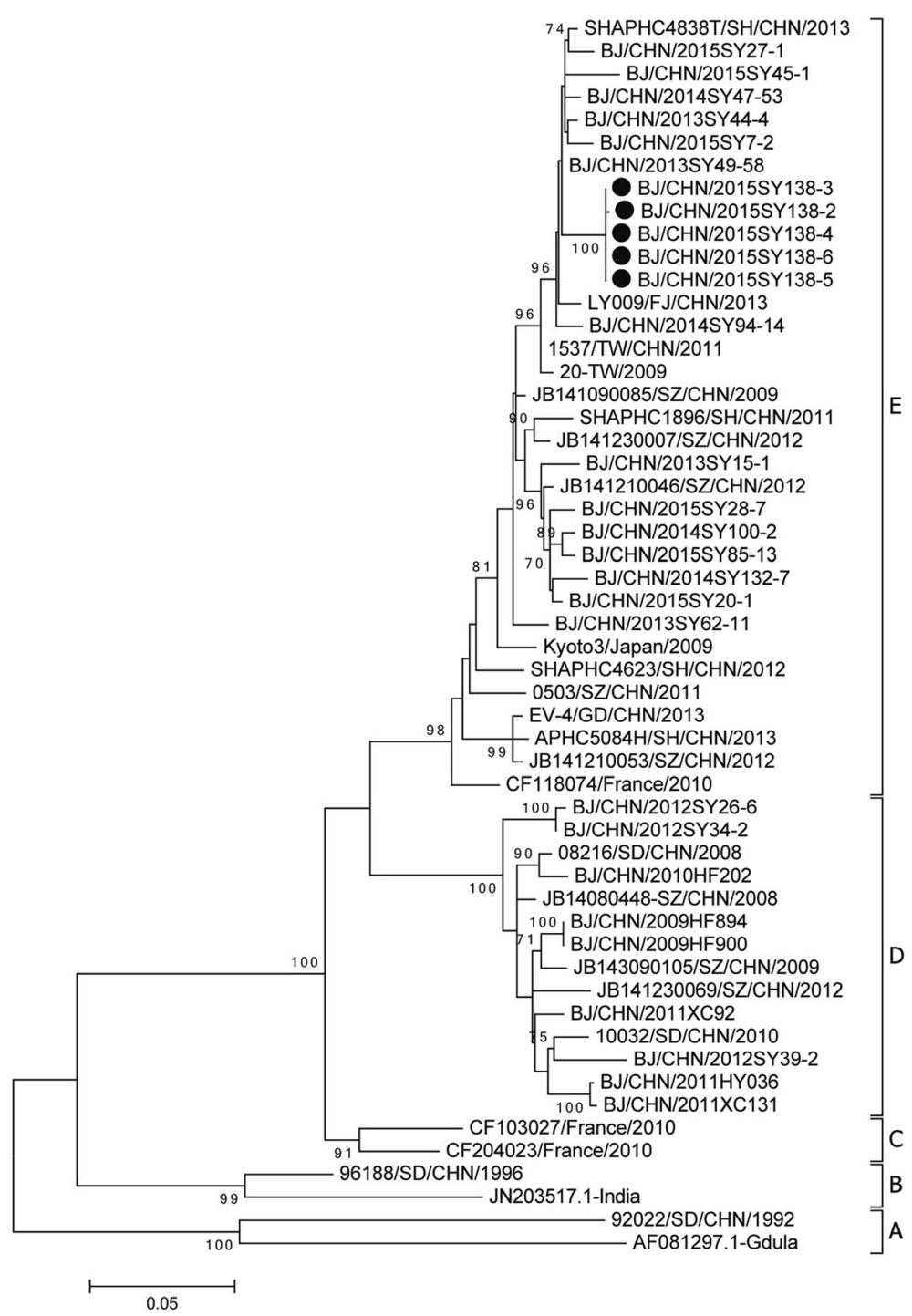

Fig. 2 Phylogenetic dendrogram based on the alignment of the complete VP1 gene sequence of CVA6 (915 bp). CVA6 strains isolated from this outbreak were indicated by a solid dot

90\% against EV-A71-associated HFMD [19]. However, these vaccines cannot protect people from suffering from HFMD caused by CVA6 [20]. In the absence of any effective vaccine or antiviral therapy, primary prevention is the most effective measure to prevent the outbreak of CVA6-associated HFMD.

A previous study has reported that different genetic background strains may cause different clinical manifestations of HFMD [21]. Confirmed by the VP1 gene sequencing, our results suggested that this outbreak was caused by the same CVA6. In this study, five out of ten CVA6-positive HFMD cases developed onychomadesis. Based on the results of this study, we proposed that the difference in clinical presentations might be correlated with the host background apart from the causative pathogen. Moreover, we found that patients with symptoms of desquamation had a 9.3-time chance of experiencing onychomadesis comparing with cases without desquamation. Combining the fact that desquamation in most cases appeared earlier than onychomadesis, we suggest that desquamation might be used as an indicator of developing onychomadesis. Based on the current situation that parents and even some physicians know little about HFMD related onychomadesis, misdiagnosis could happen. We hope that this study can provide evidence for the early treatment of HFMD and help address the concerns of the parents.

There are several limitations. First, we did not assess the effectiveness of individual control measure. Second, the data collection was telephone-based and recall bias might exist. Third, due to limited knowledge about the late symptoms of HFMD, the appearance of 
desquamation, onychomadesis or pigmentation might be underestimated.

\section{Conclusion}

In summary, the 2015 Beijing HFMD outbreak was caused by CVA6, featured with delayed symptoms. Health care providers and parents need to pay more attention and raise awareness towards the CVA6-HFMD to be able to better control and prevent future disease outbreak.

\section{Additional file}

Additional file 1: Onychomadesis distribution of finger nails affected per cases. (DOCX $21 \mathrm{~kb})$

\section{Abbreviations}

CDC: Center for Disease Control and Prevention; CRP: C-reactive protein; CVA16: Coxsackievirus A16; CVA6: Coxsackievirus A6; EV-A71: Human enterovirus 71; HFMD: Hand, foot and mouth disease; WBC: White blood cell

\section{Acknowledgments}

We thank those participants in this study who provided samples and clinical data. We also thank the staffs who work in the SCDC and local health care centers for collecting epidemiological information and samples during the execution of this study.

\section{Funding}

This work was supported by grants of key projects in the National Science \& Technology Pillar Program (2015BAI09B01). This work was also supported by the grant from Beijing Natural Science Foundation to YH (7132027) and the grant from Beijing Natural Science Foundation to JL (7164240).

\section{Availability of data and materials}

The datasets used and analyzed in this study is available upon request of author JL.

\section{Authors' contributions}

$J \mathrm{~L}$ and RZ carried out the experimental design, participated in the experiment and drafted the manuscript. They contributed equally to this study and share first authorship. DH, YD and YY participated in the experimental design, performed the data analysis, and reviewed the manuscript. LZ, YL and YY participated in the experiment and reviewed the manuscript. LJ and LC participated in the clinical data collection, analysis, and reviewed the manuscript. QW and $\mathrm{YH}$ contributed to the experimental design and coordination with District Centers for Disease Control and Prevention and provided a final review of this manuscript. All authors read and approved the final manuscript.

\section{Ethics approval and consent to participate}

This study was in compliance with the Helsinki Declaration and was approved by the Human Research Ethics Committee of Beijing CDC. Written informed consent for sample collection in this study was received from the patient's guardian.

\section{Consent for publication \\ Not applicable.}

\section{Competing interests}

The authors declare that they have no competing interests.
Received: 25 March 2018 Accepted: 13 August 2018

Published online: 21 August 2018

References

1. Ooi MH, Wong SC, Lewthwaite P, Cardosa MJ, Solomon T. Clinical features, diagnosis, and management of enterovirus 71. Lancet Neurol J. 2010:9:1097-105.

2. Abzug MJ. The enteroviruses: problems in need of treatments. J Inf Secur. 2014;68(Suppl 1):S108-14.

3. He Y, Zou L, Chong MK, Men R, Xu W, Yang H, et al. Genetic evolution of human enterovirus A71 subgenotype C4 in Shenzhen, China, 1998-2013. J Inf Secur. 2016;72:731-7

4. Hongyan $\mathrm{G}$, Chengjie M, Qiaozhi $Y$, Wenhao $H$, Juan L, Lin P, et al. Hand, foot and mouth disease caused by coxsackievirus A6, Beijing, 2013. Pediatr Infect Dis. 2014:33:1302-3.

5. Tan X, Li L, Zhang B, Jorba J, Su X, Ji T, et al. Molecular epidemiology of coxsackievirus A6 associated with outbreaks of hand, foot, and mouth disease in Tianjin, China, in 2013. Arch Virol. 2015;160:1097-104.

6. Hu YQ, Xie GC, Li DD, Pang LL, Xie J, Wang P, et al. Prevalence of Coxsackievirus A6 and enterovirus 71 in hand, foot and mouth disease in Nanjing, China in 2013. Pediatr Infect Dis J. 2015;34:951-7.

7. Han JF, Xu S, Zhang Y, Zhu SY, Wu DL, Yang XD, et al. Hand, foot, and mouth disease outbreak caused by coxsackievirus A6, China, 2013. J Inf Secur. 2014:69:303-5.

8. Xing W, Liao Q, Viboud C, Zhang J, Sun J, et al. Hand, foot, and mouth disease in China, 2008-12: an epidemiological study. Lancet Infect Dis. 2014;14:308-18.

9. The national health and Family Planning Commission. (2012) Protocol of control practice for the clustered cases and outbreaks of hand foot and mouth disease. http://www.chinacdc.cn/jkzt/crb/szkb/jszl_2275/201206/ t20120629 63852 htm. Accessed 1 June 2012

10. Ministry of Health of the People's Republic of China. Protocol of sample collection and laboratory tests for HFMD cases. http://www.chinacdc.cn/ jkzt/crb/szkb/jszl_2275/200906/t20090612_24707.htm. Accessed 2009.

11. Nix WA, Oberste MS, Pallansch MA. Sensitive, seminested PCR amplification of VP1 sequences for direct identification of all enterovirus serotypes from original clinical specimens. J Clin Microbiol. 2006:44:2698-704

12. Sinclair C, Gaunt E, Simmonds P, Broomfield D, Nwafor N, et al. Atypical hand, foot, and mouth disease associated with coxsackievirus A6 infection, Edinburgh, United Kingdom, January to February 2014. Euro Surveill. 2014; 19:20745.

13. Jacobsen L, Zimmerman S, Lohr J. Nail findings in hand-foot-and-mouth disease. Pediatr Infect Dis J. 2015;34:449-50

14. Apalla Z, Sotiriou E, Pikou O, Lefaki I, Lallas A, Lazaridou E, et al. Onychomadesis after hand-foot-and-mouth disease outbreak in northern Greece: case series and brief review of the literature. Int J Dermatol. 2015;54:1039-44

15. Li J, Sun Y, Du Y, Yan Y, Huo D, Liu Y, et al. Characterization of Coxsackievirus A6- and enterovirus 71-associated hand foot and mouth disease in Beijing, China, from 2013 to 2015. Front Microbiol. 2016:7:391.

16. Wong SS, Yip CC, Lau SK, Yuen KY. Human enterovirus 71 and hand, foot and mouth disease. Epidemiol Infect. 2010;138:1071-89.

17. Li J, Lin C, Qu M, Li X, Gao Z, et al. Excretion of enterovirus 71 in persons infected with hand, foot and mouth disease. Virol J. 2013;10:31.

18. Kar BR, Dwibedi B, Kar SK. An outbreak of hand, foot and mouth disease in Bhubaneswar, Odisha. Indian Pediatr. 2013;50:139-42.

19. Chen YJ, Meng FY, Mao Q, Li JX, Wang H, Liang ZL, et al. Clinical evaluation for batch consistency of an inactivated enterovirus 71 vaccine in a largescale phase 3 clinical trial. Hum Vaccin Immunother. 2014;10:1366-72.

20. Liu CC, Chow YH, Chong P, Klein M. Prospect and challenges for the development of multivalent vaccines against hand, foot and mouth diseases. Vaccine. 2014;32:6177-82.

21. Chen YJ, Chang SC, Tsao KC, Shih SR, Yang SL, Lin TY, et al. Comparative genomic analysis of coxsackievirus A6 strains of different clinical disease entities. PLoS One. 2012;7:e52432.

\section{Publisher's Note}

Springer Nature remains neutral with regard to jurisdictional claims in published maps and institutional affiliations. 